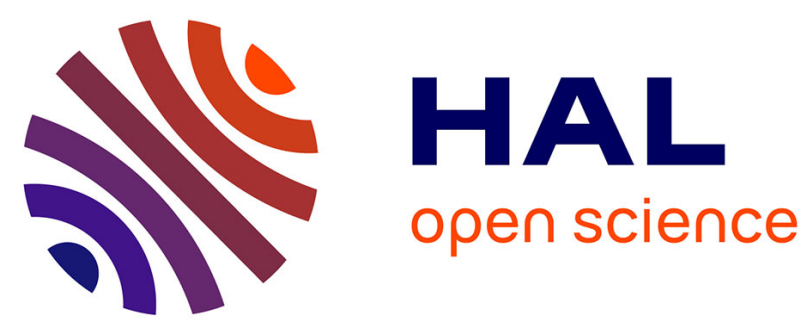

\title{
ACOUSTOÉLASTICITÉ SOUS INCIDENCE; ANISOTROPIE INDUITE, TEXTURE ET CONTRAINTES RIÉSIDUELLES
}

Rachid El Guerjouma, M. Deschamps, A. Gerard

\section{- To cite this version:}

Rachid El Guerjouma, M. Deschamps, A. Gerard. ACOUSTOÉLASTICITÉ SOUS INCIDENCE; ANISOTROPIE INDUITE, TEXTURE ET CONTRAINTES RIÉSIDUELLES. Journal de Physique IV Proceedings, 1992, 02 (C1), pp.C1-853-C1-856. 10.1051/jp4:19921186 • jpa-00251148

HAL Id: jpa-00251148 https://hal.science/jpa-00251148

Submitted on 1 Jan 1992

HAL is a multi-disciplinary open access archive for the deposit and dissemination of scientific research documents, whether they are published or not. The documents may come from teaching and research institutions in France or abroad, or from public or private research centers.
L'archive ouverte pluridisciplinaire HAL, est destinée au dépôt et à la diffusion de documents scientifiques de niveau recherche, publiés ou non, émanant des établissements d'enseignement et de recherche français ou étrangers, des laboratoires publics ou privés. 


\title{
ACOUSTOÉLASTICITÉ SOUS INCIDENCE ; ANISOTROPIE INDUITE, TEXTURE ET CONTRAINTES RÉSIDUELLES
}

\author{
R. EL GUERJOUMA, M. DESCHAMPS* et A. GERARD* \\ Laboratoire de Traitement du Signal et Ultrasons, URA CNRS 1216, Bat 502, INSA de Lyon, \\ F-69621 Villeurbanne cedex, France \\ ${ }^{*}$ Laboratoire de Mécanique Physique, URA CNRS 867, Université de Bordeaux I, F-33405 Talence \\ cedex, France
}

\begin{abstract}
In this work, we discuss the limits of the acoustoelastic isotropic model. We examine experimentally the influence on the acoustoelastic behaviour of the plastic strain and recrystallization heat treatment to appreciate the contribution on this behaviour of the texture, residual and applied stresses
\end{abstract}

\section{I - Introduction}

Un solide homogène et isotrope soumis à des contraintes statiques et homogènes perd son isotropie dans le sens où des ondes ultrasonores superposées aux contraintes statiques exibent un comportement anisotrope. Ce phénomène connu sous le nom d'acoustoélasticité est à la base de nombreuses téchniques d'analyse des contraintes et de détermination de constantes d'élasticité d'ordre trois.

Les matériaux réels sont rarement isotropes et les processus de fabrication ou de mise en forme se traduisent par une texture et la présence de contraintes résiduelles. Ainsi l'hypothèse d'isotropie communément admise apparait comme extrêmement restrictive. Plus surprenant, partant des mêmes hypothèses (matériau homogène et isotrope, contraintes homogènes et uniaxiales, propagation de modes propres), des expressions acoustoélastiques différentes sont établies par plusieurs auteurs. Une étude comparative récente [1] entre plusieurs modèles a aboutit également à ce constat. De ce fait, les constantes d'élasticité disponibles dans la littérature sont souvent très différentes pour un même matériau isotrope, d'une étude à l'autre, ce qui remet en question les fondements et la validité de ces modèles.

Dans le présent article nous nous proposons de mettre en évidence expérimentalement les limites du modèle isotope, d'apprécier les influences de la contrainte homogène appliquée, des contraintes résiduelles et de la texture sur le comportement acoustoélastique à l'aide d'un dispositif à immersion permettant des mesures de vitesses à incidence variable, en fonction d'une contrainte appliquée . Dans un premier temps, les résultats de l'étude théorique de l'anisotropie induite par une contrainte uniaxiale au sein d'un matériau isotrope sont rappelés. Puis les limites de ce modèle isotrope sont mises en évidence sur un alliage d'aluminium et sur du cuivre. Les influences respectives de la texture et des contraintes résiduelles sont étudiées en considérant les effets d'une recristallisation et d'une déformation plastique sur les réponses acoustoélastiques.

\section{II - Acoustoẻlasticité sous incidence - Théorie isotrope}

L'élasticité linéaire est décrite par la loi de Hooke généralisée, son écriture isotherme est :

(1)

$$
\sigma_{\mathrm{ij}}=\mathbf{C}_{\mathrm{ijkl}} \varepsilon_{\mathrm{kJ}} \text {, }
$$

où $\sigma_{\mathrm{ij}}\left(\varepsilon_{\mathrm{kl}}\right)$ sont les éléments des tenseurs des contraintes (déformations) et $\mathrm{C}_{\mathrm{ijkl}}$ les constantes d'élasticité d'ordre 2. Le comportement non linéaire élastique résulte de la contribution à la loi de comportement des 
constantes d'élasticité d'ordre supérieur à 2. Cette contribution est l'écart par rapport à la loi de Hooke. En effet pour un matériau hyperélastique, l'énergie de déformation par unité de volume peut s'écrire en se limitant à l'ordre 3:

(2)

$\Sigma=\frac{1}{2} C_{i j k} E_{i j} E_{k l}+\frac{1}{6} C_{i j k l m n} E_{i j} E_{k l} E_{m n}, C_{i j k j l m n}$ constantes d'élasticité d'ordre $3, E_{i j}$ déformations.

Le comportement élastique non linéaire des matériaux peut-être déterminé à partir de l'étude de l'interaction ondes ultrasonores - contraintes mécaniques statiques. Ainsi le developpement de la théorie de l'acoustoélasticité est à la base un problème de superposition d'une petite perturbation ultrasonore à une contrainte statique. Désignons par w le vecteur déplacement attaché à l'onde ultrasonore et cherchons les solutions de l'équation d'équilibre de l'état perturbé sous forme d'ondes planes se propageant dans la direction $\mathbf{n}$ avec la célérité $V$. On est conduit à résoudre le problème aux valeurs propres[2]:

$$
\left[A_{i j}-\rho_{0} V^{2} \delta_{i j}\right] w_{j}=0,
$$

$A_{i j}$ est le tenseur acoutoélastique fonction de la direction de propagation et des constantes d'élasticité d'ordre 2 et 3 , $\rho_{0}$ est la densité au repos[3]. Pour un matériau isotrope, les constantes d'élasticité $\mathrm{C}_{\mathrm{ijkl}}$ au nombre de 2 et $C_{\mathrm{ijklmn}}$ au nombre de 3 sont fonction respectivement des coefficients d'ordre 2 de Lamé $\lambda$ et $\mu$ et d'ordre $3: v_{1} v_{2} v_{3}$ définis par Toupin et Bernstein où $1, \mathrm{~m}, \mathrm{n}$ définis par Murnagan, les 2 notations sont équivalentes[3]. Considérons le cas où la charge est appliquée suivant la direction 1, la propagation ayant lieu dans le plan $(1,3)$ dans la direction $\mathbf{n}(\sin \theta, 0, \cos \theta), \theta=(3, \mathbf{n})$ est l'angle de transmission. La résolution de (3) fournit l'expression des vitesses de propagation dans le plan $(1,3)$ [2].

$$
\mathrm{V}_{1}(\theta)-\mathrm{V}_{10}=\mathrm{L}(\theta) . \sigma ; \mathrm{V}_{\mathrm{t}}(\theta)-\mathrm{V}_{\mathrm{t} 0}=\mathrm{T}(\theta) . \sigma
$$

$V_{10}=\left[(\lambda+2 \mu) / \rho_{0}\right]^{1 / 2}$ et $V_{t 0}=\left[\mu / \rho_{0}\right]^{1 / 2}$ sont les vitesses longitudinale et transversale à l'état non chargé, indépendantes de $\theta$ (isotropie) et $\mathrm{L}(\theta)$ et $\mathrm{T}(\theta)$ réponses acoustoélastiques isotropes[2]

Lorsque la direction de propagation est confondue avec celle de la contrainte uniaxiale on retrouve les expressions établies par Danthez[3] dans le cas des milieux cylindriques tendus. Les expressions analytiques des vitesses montrent une anisotropie induite par les contraintes. Il est à remarquer que les différentes approches théoriques partant des mêmes hypothèses aboutissent toutes à une anisotropie induite mais donnent souvent des expressions analytiques diffèrentes pour les variations de vitesses. Une étude comparative récente de certaines modélisations isotropes et de leurs validations expérimentales[1] a aboutit à la même constatation et conclut à une erreur dans les developpements de Hughes et Kelly [4], développements dont nombre d'auteurs se sont inspirés. Ceci montre qu'au dela du fait que le modèle isotrope soit très restrictif comme nous allons le voir puisque les matériaux réels sont texturés, les developpements de certains modèles notament celui de Hughes et Kelly sont à revoir de manière à lever les ambiguités constatées.

\section{III - Résultats expérimentaux : Alliage d'aluminium}

Une installation complexe mise au point au Laboratoire de Mécanique Physique, composée d'un banc de caractérisation ultrasonore associé à une machine de traction de faible volume, permet d'accéder aux vitesses de propagation des ondes au sein du matériau en fonction de l'angle d'incidence (ou de transmission) et de la contrainte appliquée [2]. La méthode utilisée pour la détermination des vitesses est fondée sur la mesure du temps de vol par corrélation-filtrage [6]. Les mesures sont effectuées par transmission en régime impulsionnel autour de la fréquence centrale de $10 \mathrm{MHz}$. Le bain est thermostaté à $25^{\circ} \mathrm{C}$. Dans les conditions précitées la méthode par corrélation-filtrage offre une précision sur la mesure du temps de vol de l'ordre de la nanoseconde. La variation de l'épaisseur acoustique en fonction de la charge est prise en compte. Elle est évaluée à incidence normale à partir de l'analyse des échos multiples. La vitesse a été moyennée sur 10 mesures, ce qui assure une précision de l'ordre du mètre par seconde.

L'alliage d'aluminium étudié est de l'AU4G. Les échantillons testés sont des éprouvettes de traction classiques (longueur $90 \mathrm{~mm}$, section $50 * 15$ ). La figure 1 présente la variation de vitesse en fonction de la charge pour différentes directions de propagation. Les réponses acoutoélastiques sont linéaires quelle que soit l'incidence. Ceci semble en accord avec la théorie (relation 4). Seulement, la vitesse à charge nulle dépend de la direction de propagation (figure 2, éprouvette non recuite), ce que le modèle isotrope occulte. Sur la figure $1 \mathrm{n}$ ' est pas représenté $: \mathrm{V}_{1}(\sigma, \theta)-\mathrm{V}_{10}$ du modèle isotrope mais $\mathrm{V}_{1}(\sigma, \theta)-\mathrm{V}_{1}(0, \theta)$. 
$V_{1}(0, \theta)$ étant la vitesse sous incidence, à contrainte nulle dont on peut constater l'évolution en fonction de $\theta$ sur la figure 2 (éprouvette non recuite). Cette évolution résulte donc des effets combinés de la texture et des contraintes résiduelles. La variation associée est de plus de $100 \mathrm{~m} / \mathrm{s}$ pour certaines directions de propagation et est nettement supérieure à celle provoquée par la contrainte appliquée (Figure 1). De manière à se rapprocher au mieux de l'hypothèse d'isotropie, nous faisons subir un traitement thermique à l'échantillon (recuit à $500^{\circ} \mathrm{C}$ pendant 5 heures suivi d'un refroidissement lent de 12 heures). L'hypothèse d'isotropie est alors satisfaisante (figure 2). Sur l'éprouvette recuite, les réponses acoustoélastiques ont été relevées. La figure 3 rend plus aisée la comparaison des réponses acoustoélastiques entre les états recuit (sensiblement isotrope) et non recuit (texturé). Les courbes réprésentatives de ces réponses présentent globalement la même évolution. Ainsi ce traitement s'il modifie la structure du matériau, relaxe les contraintes résiduelles, n'altère pas de façon notable les réponses acoutoélastiques de celui-ci.

Ce résultat rend compte du couplage et de la prédominance des effets de texture et de contraintes résiduelles sur les effets de contraintes appliquées. Il apparait ainsi trés hasardeux de vouloir estimer les constantes d'élasticité du troisième ordre en utilisant le modèle isotrope sur des matériaux texturés[5]. Par contre sur le matériau recuit le modèle isotrope peut être utilisé. A partir de l'expression de la réponse acoustoélastique en ondes longitudinales $L(\theta)$, les constantes d'élasticité d'ordre 3 peuvent être estimées en utilisant les valeurs expérimentales des réponses acoustoélastiques et une méthode classique d'optimisation au sens des moindres carrés[2]. Les valeurs identifiées pour l'échantillon d'AU4G étudié sont présentées sur le tableau 1 et comparées à celle obtenues par Chatelier [7].

\begin{tabular}{|l|c|c|c|}
\cline { 2 - 4 } \multicolumn{1}{c|}{} & $v 1$ & $v 2$ & $v 3$ \\
\hline Optimisation & 149 & -33 & -216 \\
\hline Chatelier & -78 & -264 & -629 \\
\hline
\end{tabular}

tableau $1\left(v_{i}\right.$ en GPa $)$

Les différences constatées tiennent certainement des traitements différents subis par les échantillons, mais il est à remarquer que les expressions théoriques des vitesses établies par Chatelier, pour un matériau isotrope se ramènent à celles de Hughes et Kelly dont le modèle est mis en cause comme cela a été mentionné plus haut.

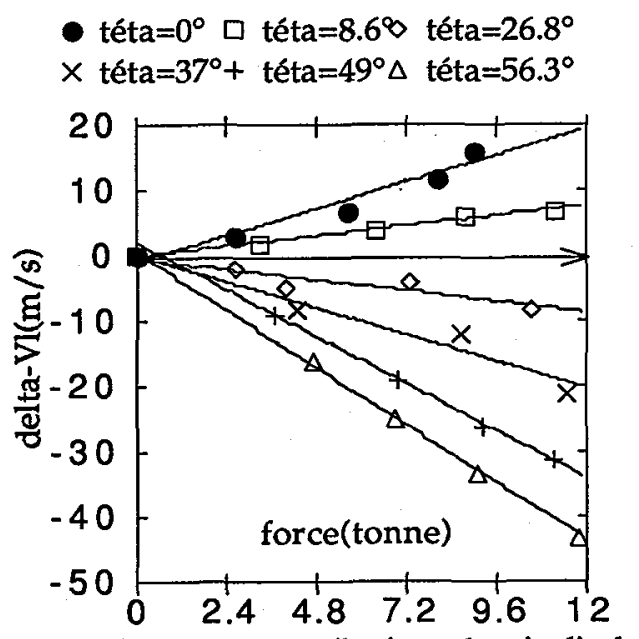

Figurel: réponses acoustoélastiques longitudinales

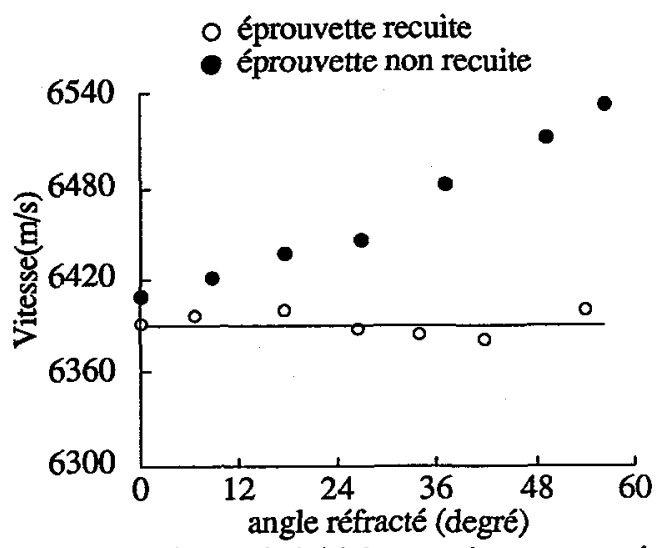

Figure 2: anisotropie initiale - recuit et non recuit

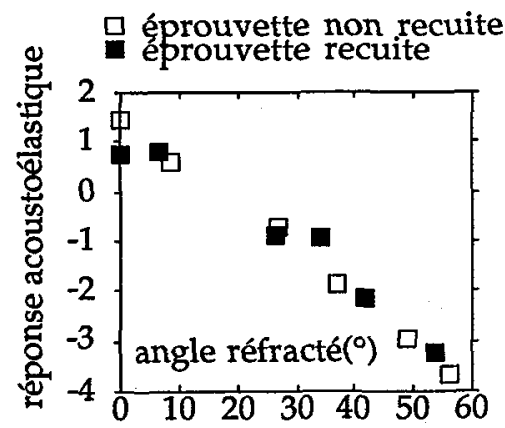

Figure 3: réponse acoustique - recuit et non recuit 


\section{Cuivre : Influence d'une déformation plastique \\ Pour cette nouvelle application, les essais sont} réalisés sur des éprouvettes de traction, en cuivre pur, de longueur $90 \mathrm{~mm}$ et de section $50 * 5$. Les réponses acoustoélastiques sont enregistrées avant et après plastification, de manière à apprécier l'influence de la déformation plastique et ainsi des contraintes residuelles sur le comportement acoustoélastique, celles-ci étant définies comme des contraintes statiques existant dans un état d'équilibre mécanique en l'absence d'efforts et de moments extérieurs. Les réponses acoutoélastiques avant plastification sont non-linéaires, de forme parabolique pour toutes les incidences et pour les deux modes propagés. Sur la figure 4 sont présentées les réponses en ondes longitudinales pour différentes incidences. On observe des variations similaires en ondes transversales. Ceci est en accord avec les résultats de Fukuoka et Toda [5] qui se sont limités à l'incidence normale $(\theta=0)$. Ces résultats ne correspondent pas du tout au prévision du modèle isotrope qui prévoit des réponses linéaires.

Les effets de contraintes appliquées sont faibles par rapport aux effets de texture, comme on peut le constater par une simple observation de l'ordonnée à l'origine de la figure 4.

Après plastification les réponses acoustoélastiques tant pour les ondes longitudinales que transversales, restent paraboliques et leur forme évolue peu comme on peut le constater sur la figure 5.

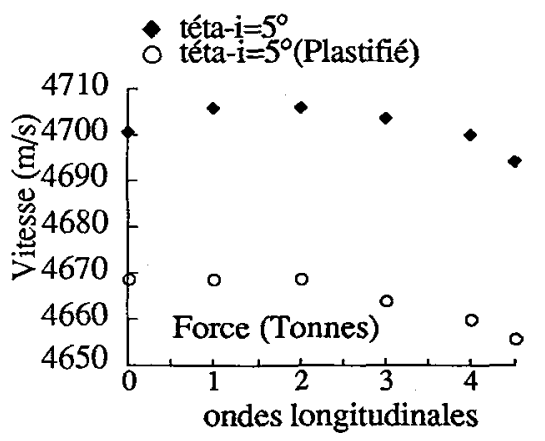

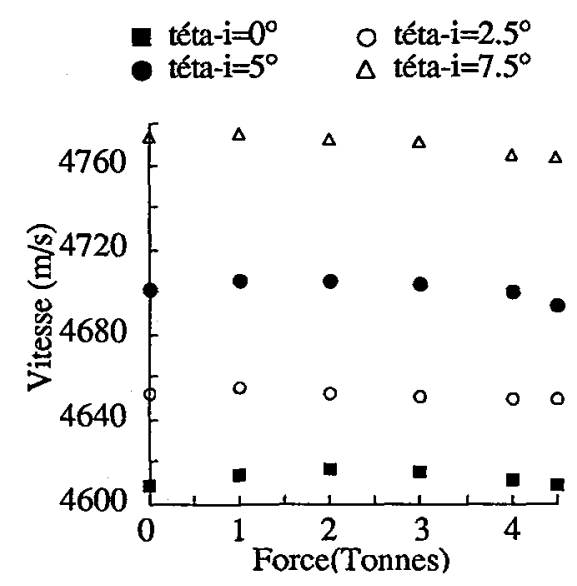

figure 4 : anisotropie initiale et réponses acoustoélastiques : ondes longitudinales

Figure 5 : réponses acoustoélastique avant et aprés plastification

La variation de vitesse entre les deux états, plastifié et non plastifié, se ramène à une translation importante pour les ondes longitudinales, de l'ordre de $30 \mathrm{~m} / \mathrm{s}$, moins pour les transversales. Les effets de contraintes résiduelles sont donc comparables à ceux des contraintes appliquées.

Ainsi ce travail a permis d'étudier et de discuter les influences sur le comportement acoustoélastique sous incidence d'une part d'un traitement thermique de recristallisation et d'une déformation plastique d'autre part, dans le but d'apprécier les effets respectifs de la texture, des contraintes résiduelles et appliquées sur ce même comportement.

\section{Bibliographie}

[1] Kheder A. and De Halleux B., Review of Propers in Q.N.D.E., Brunswick, USA July 1991

[2] El Guerjouma R., Thèse de l'université de Bordeaux I (1989)

[3] Danthez J.M., Thèse de l'Université de Bordeaux I (1988)

[4] Hughes D.S. and Kelly J.L., Physical Review, 92 (5), 1145-1149 (1953)

[5] Fukuoka H. Toda H.- Arch. of Mech., 29 (5), 673-686 (1977)

[6] Roux J., Hosten B., Castagnede B., Deschamps M., Revue Phys. Appl. 20, 351-358 (1985)

[7] Chatelier J.Y., Thèse de doctorat, ENSAM, Paris (1987) 